\title{
An Unusual Presentation of CNS Histoplasmosis Mimicking Metastatic Cancer
}

\author{
Prashanth Moku, Shady Piedra Abusharar, Fahad M Khalid, Rashmi Banjade and Hyma Polimera* \\ Penn State College of Medicine, USA
}

Submission: September 10, 2019; Published: September 18, 2019

*Corresponding author: Hyma Polimera Penn State College of Medicine, Penn State Health Milton S Hershey Medical center, 500 university drive, Hershey, PA 17033, USA

\begin{abstract}
Histoplasmosis capsulatum is a thermally dimorphic fungus that is known to cause histoplasmosis. Most histoplasma infections are mild or asymptomatic in immunocompetent individuals but affected individuals often present with manifestations of an inflammatory response to this infection. We report a case of disseminated histoplasmosis complicated by CNS and adrenal gland involvement.
\end{abstract}

Keywords: Histoplasma capsulatum; CNS histoplasmosis; fungus

\section{Introduction}

Histoplasma Capsulatum is a thermally dimorphic fungus that is known to cause histoplasmosis. Inhalation of Histoplasma microconidia into alveolar spaces transforms them into yeasts. Most histoplasma infections are mild or asymptomatic in immunocompetent individuals. However, affected individuals often present with manifestations of an inflammatory response to this infection. Patients often present with bone marrow suppression (pancytopenia) and elevated liver enzymes [1]. Clinical evaluation reveals a flu-like illness with findings of fever, headache, myalgia, dyspnea, chest pain and infection localized to the lungs [2]. Additional manifestations can include involvement of adrenal glands, central nervous system, and skin [3]. Herein, we present a case of disseminated histoplasmosis complicated by CNS and adrenal gland involvement.

\section{Case Description}

A 62-year-old Caucasian man with history of coronary artery disease and bypass graft presented with progressive fatigue, anorexia, and a 75-lb weight loss over a 3-month period. Diagnostic workup by his primary care physician had included CT scans of chest and abdomen/pelvis with contrast that showed bilateral granular ground-glass opacities in lungs as well bilateral adrenal masses and hepatosplenomegaly. Subsequent brain MRI then revealed multiple enhancing lesions, which were concerning for metastasis (Figure 1). Abdominal MRI also revealed a $39.6 \mathrm{~mm} \times 33 \mathrm{~mm}$ left adrenal mass that was concerning for metastasis given the patient's associated complaints of splenomegaly and pancytopenia (Figure 2). He was then referred to an oncologist, who proceeded with outpatient workup for presumed malignancy, but because of progressive fatigue, hypotension and hypoglycemia, he was admitted to an outside hospital. Later, he was transferred to our institution for further management. On admission, he was noted have pancytopenia and hypercalcemia $(\mathrm{Ca}=11.7)$.

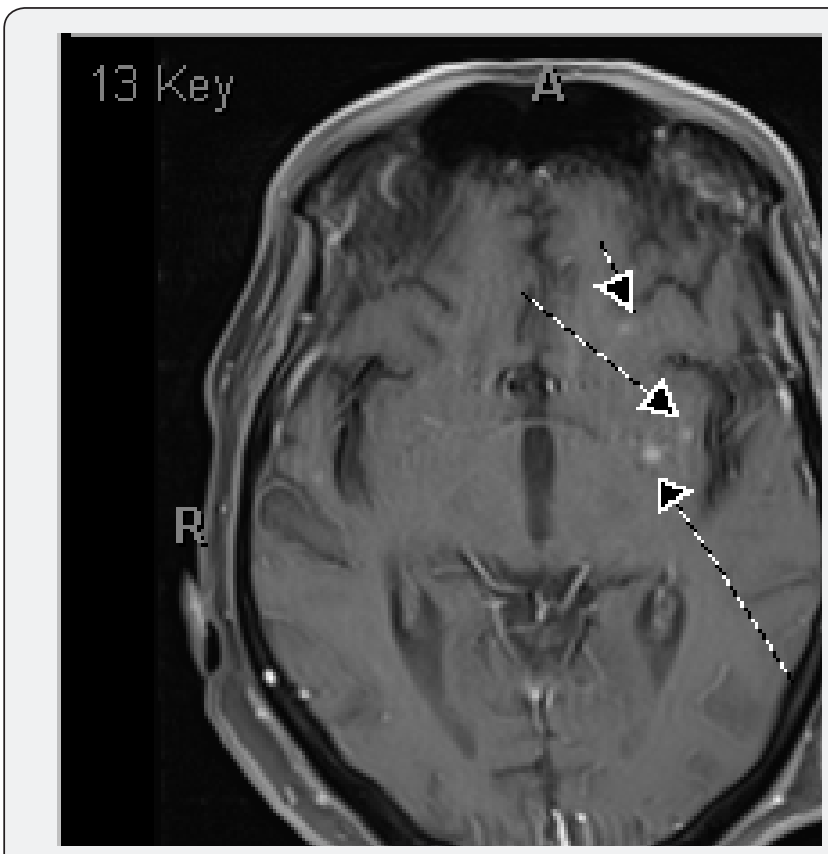

Figure 1: Brain MRI reveals numerous sub centimeter enhancing lesions scattered throughout the supratentorial brain parenchyma, with most prominent lesions in left inferior frontal lobe, findings compatible with intracranial metastatic disease. 


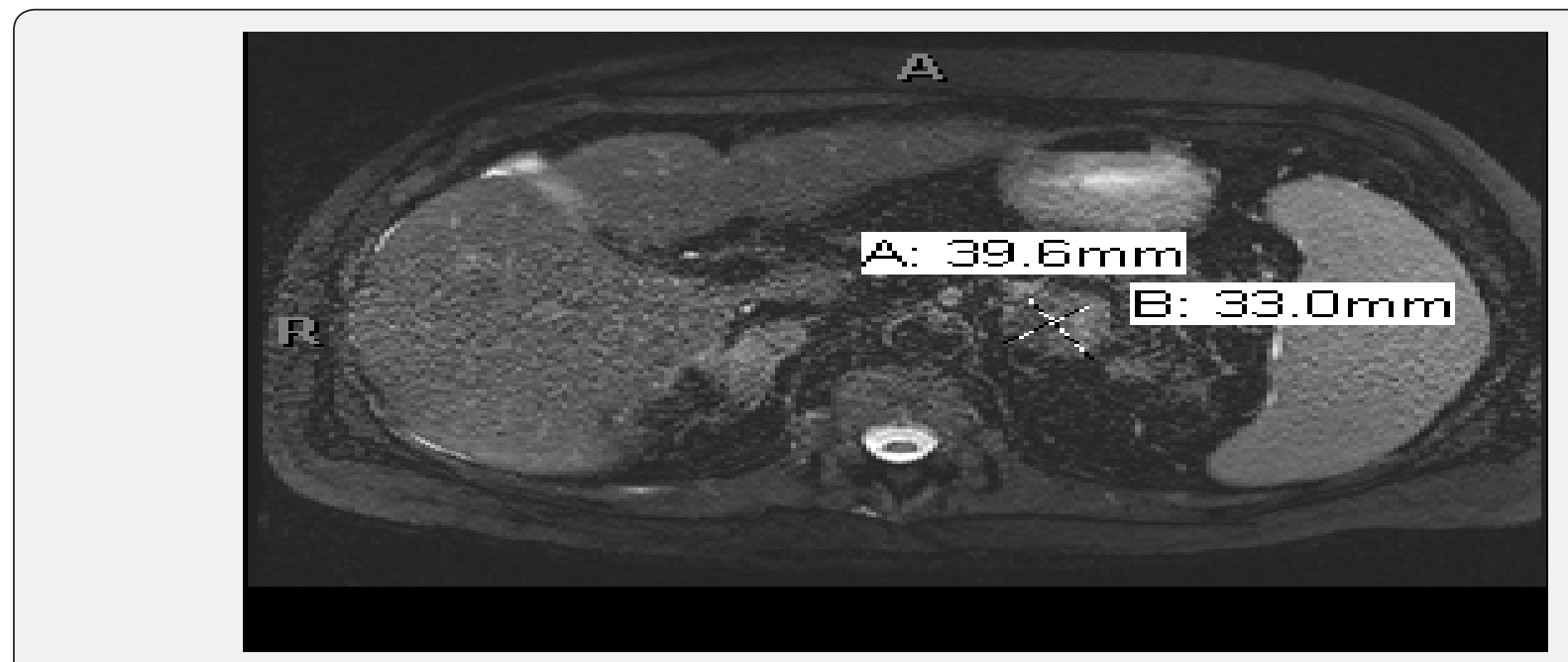

Figure 2: Abdominal MRI with contrast reveals heterogenous left adrenal mass measuring $39.6 \mathrm{~mm} \times 33 \mathrm{~mm}$ with restricted diffusion within the mass. Findings inconsistent with benign adenoma; given patient's pancytopenia and splenomegaly, metastatic disease was considered.

Subsequent biopsy of adrenal gland showed nonencapsulated small yeasts, suggestive of either Histoplasma or Cryptococcus. He was started on IV amphotericin B for possible histoplasmosis while waiting for further diagnostic work-up. His serum and urine Histoplasma Ag returned positive with high titers, adrenal tissue culture grew Histoplasma Capsulatum and his blood cultures for fungus also grew Histoplasma Capsulatum after several days. Lumbar puncture also revealed positive CSF Histoplasma antigen, though the level was below level of quantification. His immunological work-up was unremarkable with non-reactive HIV test. Initial CD4 count was low at 166 (39\%), but it quickly returned to normal after treatment with IV Amphotericin B suggesting bone marrow suppression from Histoplasmosis itself rather than an underlying immune disorder. He was treated with eight weeks of IV amphotericin B and transitioned to oral Itraconazole for a prolonged period of time that he continues to remain on with good response.

\section{Discussion}

Histoplasma Capsulatum is a thermally dimorphic fungus which infects both immunocompetent and immunocompromised hosts [4]. It is endemic to the Ohio and Mississippi River Valleys in the United States and parts of Latin America, Africa, and Asia [5,6]. H. Capsulatum is commonly found in the soil and contaminated material of bat or bird guano. Spread of the disease occurs via inhalation of spores from disturbed guano or soil [5]. In immune-competent persons, spore inhalation commonly results in an asymptomatic infection [5]. Exposure in immune-incompetent patients, due to its mode of transmission via spore inhalation, histoplasmosis most commonly manifests in the lungs, ranging from pneumonitis to severe acute respiratory distress syndrome (ARDS). The yeast form is disseminated via macrophages as they travel throughout the body [6]. CNS involvement in histoplasmosis is either a manifestation of disseminated infection or an isolated focal infection. CNS involvement has been reported in 5-10\% of cases of disseminated histoplasmosis, but isolated CNS histoplasmosis is rare, and the exact pathophysiologic mechanism is not known. Although CNS histoplasmosis is increased in individuals with impaired cellular immunity, about $20-30 \%$ of patients with this condition are immunocompetent [4].

The clinical features, appropriate diagnostic testing, and treatment regimens recommended for the evaluation, and management of CNS histoplasmosis are based on case reports, and small case series of patients [7]. The most common manifestations of CNS histoplasmosis consist of chronic meningitis and hydrocephalus. Less common presentations include acute meningitis, encephalitis, small ring enhancing lesions throughout the brain and spinal cord, brain abscesses and stroke due to infected emboli. In rare cases, patients with CNS histoplasmosis may present with cachexia and hypercalcemia [6]. Symptoms range from headache, mental status changes, cranial nerve palsies, behavioral changes and ataxia [4]. Morbidity, and mortality appear to be high in patients with CNS histoplasmosis with high rates of relapse [7].

The diagnosis of CNS histoplasmosis can be challenging. Multiple tests for diagnosis of $\mathrm{H}$. capsulatum in the CNS are necessary due to the variability in symptoms and the number of false-negative tests. Culturing of the CSF or CNS parenchymal tissue is the gold standard for diagnosis of CNS histoplasmosis. Antigen detection in CSF culture has a sensitivity of $38 \%$ and a specificity of $98 \%$. Additional tests for $\mathrm{H}$. capsulatum polysaccharide antigen (HPA) in CSF, urine, or serum can be performed to aid in the diagnosis and monitor response to therapy. The sensitivity of HPA in the CSF is $38 \%$ in all patients and $67 \%$ in patients with AIDS. However, the sensitivity of Histoplasma antigen in Urine and Serum is $71 \%$, and $38 \%$ respectively [6,8]. Antibody testing in CSF and serum has 80 to $89 \%$ and $92 \%$ sensitivity, respectively, although these tests 
are susceptible to cross-reactivity leading to false positives. Serologic testing is especially difficult with AIDS patients and often provides false negatives even with an active infection. In addition, positive CSF culture results are similar to results seen in other fungal and tuberculous meningitis. Therefore, other tests must be performed. MRI often shows single or multiple ring-enhancing lesions in CNS histoplasmosis. However, ringenhancing lesions can also be indicative of an abscess, a necrotic tumor, subdural and epidural empyema or toxoplasmosis. Histoplasmosis should be considered if symptoms correlate to that of the infection or if the patient has had exposure to an endemic area [6].

Optimal treatment for CNS histoplasmosis is unclear. No prospective studies with an evidence-based approach are available. Antifungal therapy is recommended for up to 12 months in immunocompetent patients. Despite appropriate therapy there is risk of failure and relapse which warrants long term suppressive therapy. Based upon expert opinion and the limited available data, IDSA (Infectious Diseases Society of America) guidelines recommend liposomal amphotericin B at a dose of $5 \mathrm{mg} / \mathrm{kg}$ daily for a total of $175 \mathrm{mg} / \mathrm{kg}$ given over 4-6weeks followed by itraconazole $200 \mathrm{mg} 2-3$ times daily for at least one year and until resolution of CSF abnormalities including HPA $[4,9]$.

\section{Conclusion}

In conclusion, clinicians should have a low threshold for considering CNS histoplasmosis as a cause for brain lesions. CNS histoplasmosis is a rare condition but requires prompt diagnosis in order to initiate appropriate treatment regimen. Our case depicts a patient who initially presented with concerns for metastatic disease in setting of hypercalcemia, pancytopenia, significant weight loss and generalized weakness. Diagnostic testing further revealed multiple enhancing lesions in brain and bilateral adrenal masses, which was strongly suspicious of malignancy. A subsequent adrenal gland biopsy was crucial in demonstrating multiple yeasts and thus, aiding in diagnosis.
In the context of the aforementioned biopsy results and the typical findings of granulomatous involvement of histoplasmosis presenting with hypercalcemia, there was strong evidence to support a diagnosis of disseminated histoplasmosis. Our patient did not have any significant risk factors, he was a normal host with no underlying immunodeficiency disorder, and he did not have significant exposure history like spelunking or exposure to bats or bird droppings. Overall, we present a case of disseminated histoplasmosis with CNS and adrenal involvement in an immunocompetent host with a unique presentation as disseminated histoplasmosis with enhancing CNS lesions.

\section{References}

1. Assi MA, Sandid MS, Baddour LM, Roberts GD, Walker RC (2007) Systemic Histoplasmosis: A 15-Year Retrospective Institutional Review of 111 Patients. Medicine (Baltimore) 86(3): 162-169.

2. Khasawneh FA, Ahmed S, Halloush RA (2013) Progressive disseminated histoplasmosis presenting with cachexia and hypercalcemia. Int J Gen Med 6: 79-83.

3. Kauffman CA (2007) Histoplasmosis: a Clinical and Laboratory Update. Clin Microbiol Rev 20(1): 115-132.

4. Ramireddy S, Wanger A, Ostrosky L (2012) An instructive case of CNS histoplasmosis in an immunocompetent host. Med Mycol Case Rep 1(1): 69-71.

5. Nguyen FN, Kar JK, Zakaria A, Schiess MC (2013) Isolated Central Nervous System Histoplasmosis Presenting with Ischemic Pontine Stroke and Meningitis in an Immune-Competent Patient. JAMA Neurol 70(5): 638-641.

6. Hariri OR, Minasian T, Quadri SA, Dyurgerova A, Farr S, et al. (2015) Histoplasmosis with Deep CNS Involvement: Case Presentation with Discussion and Literature Review. Journal of Neurological Surgery Reports 76(1): e167-e172.

7. Wheat J, Myint T, Guo Y, Kemmer P, Hage C, et al. (2018) Central nervous system histoplasmosis Multicenter restrospective study on clinical features, diagnostic approach and outcome of treatment. Medicine (Baltimore) 97(13): e0245.

8. Eid AJ, Leever JD, Husmann K (2015) Compartmentalized Histoplasma capsulatum Infection of the Central Nervous System. Case Rep Infect Dis 2015: 581415.

9. Trofa D, Nosanchuk JD (2012) Histoplasmosis of the Central Nervous System. Journal of Neuroparasitology 3(235594): 7.

\section{Your next submission with Juniper Publishers will reach you the below assets}

- Quality Editorial service

- Swift Peer Review

- Reprints availability

- E-prints Service

- Manuscript Podcast for convenient understanding

- Global attainment for your research

- Manuscript accessibility in different formats

( Pdf, E-pub, Full Text, Audio)

- Unceasing customer service

Track the below URL for one-step submission https://juniperpublishers.com/online-submission.php 